\title{
Spectral Properties of Dyes with Interfragmental Charge Transfer: Solvatochromism and Solvatofluorochromism of 2-(3-Coumaroyl)-benzopyrylium Perchlorates
}

\author{
Edward V. Sanin, ${ }^{1,2}$ Alexander I. Novikov, ${ }^{3}$ and Alexander D. Roshal ${ }^{1}$ \\ ${ }^{1}$ Research Institute of Chemistry, V. N. Karazin Kharkiv National University, Svobody Square 4, Kharkiv 61022, Ukraine \\ ${ }^{2}$ State Scientific Institution "Institute for Single Crystals", 60 Lenina Avenue, Kharkiv 61001, Ukraine \\ ${ }^{3}$ Guard Department, National Technical University "Kharkiv Polytechnic Institute", 192 Poltavskii Shlyakh Street, \\ Kharkiv 61034, Ukraine
}

Correspondence should be addressed to Alexander D. Roshal; alexandre.d.rochal@univer.kharkov.ua

Received 26 September 2014; Revised 29 November 2014; Accepted 29 November 2014; Published 23 December 2014

Academic Editor: Hicham Fenniri

Copyright (C) 2014 Edward V. Sanin et al. This is an open access article distributed under the Creative Commons Attribution License, which permits unrestricted use, distribution, and reproduction in any medium, provided the original work is properly cited.

\begin{abstract}
The paper deals with the spectral properties of dyes with interfragmental charge transfer, in particular, derivatives of 2-(3coumaroyl)benzopyrylium (CBP) perchlorates. The positions of long-wavelength absorption and emission bands, quantum yields, and lifetimes of fluorescence were measured, mostly in aprotic solvents. Regression analysis of the relationship between the spectral characteristics of $\mathrm{CBP}$ and the main solvent parameters (polarity, polarisability, nucleophilicity, and electrophilicity) was carried out. It was found that the characteristics of CBP depend mainly on two solvent parameters-polarity and/or nucleophilicity. An increase in these parameters results in a hypsochromic shift of absorption bands and a decrease of lifetimes and fluorescence intensity. The positions of the emission bands can demonstrate either hypsochromism or bathochromism, depending on the nature of the substituents. The solvatofluorochromic effects are not as strong as the solvatochromic ones. We believe that the spectral behaviour of CBP can be explained by cation depolarisation and by a decrease in the stability of nucleophilic complexes with solvent molecules that is associated with interfragmental charge transfer following excitation, relaxation, and radiative deactivation of the excited state.
\end{abstract}

\section{Introduction}

Ionic chromophores and fluorophores are commonly used as probes for estimating the electrical potential of biological membranes [1,2], as well as the solvent nucleophilicity [3] and hydration level of biological and model samples [4].

There is a very promising class of probes, the molecules of which contain two fragments referred to as "antenna" and "acceptor." The signal of an "antenna" fragment is usually due to the appearance of an electrical charge (e.g., during complex formation with a metal ion) or due to mediuminduced charge redistribution (as a result of the formation of nucleophilic complexes or hydrogen bonds with solvent molecules) [5].

A change to the "antenna" state affects the electronic structure of the "acceptor" fragment, which causes its spectral properties to change. Signal transfer from the "antenna" to the "acceptor" can take place by means of dipole-dipole resonance, field effect, or interfragmental charge transfer (ICT) $[5,6]$.

Here we have a curious case when the molecular orbitals responsible for UV-Vis spectrum formation are localised not only on the "acceptor," but also on the "antenna." Then, the changes in the absorption spectra of such probes may be due to changing parameters not only of local electronic transitions of the "acceptor," but also of local "antenna" and ICT transitions. Correspondingly, the nature of the emission bands can also differ.

Such a structure is typical of 2-(2-oxo-2H-3-chromenyl)chromenium (or 2-(3-coumaroyl)benzopyrylium, CBP) derivatives, in which positively charged benzopyrylium (BP) and neutral coumaroyl (C) fragments act as the "antenna" and 


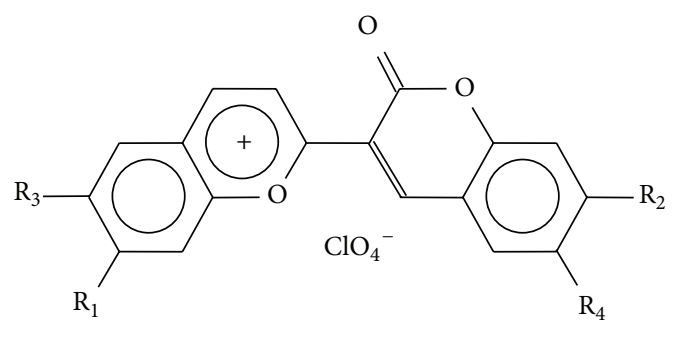

\begin{tabular}{lcccc}
\hline & $\mathrm{R}_{1}$ & $\mathrm{R}_{2}$ & $\mathrm{R}_{3}$ & $\mathrm{R}_{4}$ \\
\hline I & $\mathrm{H}$ & $\mathrm{H}$ & $\mathrm{H}$ & $\mathrm{H}$ \\
II & $\mathrm{OH}$ & $\mathrm{H}$ & $\mathrm{C}_{6} \mathrm{H}_{13}$ & $\mathrm{H}$ \\
III & $\mathrm{H}$ & $\mathrm{OH}$ & $\mathrm{H}$ & $\mathrm{C}_{6} \mathrm{H}_{13}$ \\
IV & $\mathrm{OH}$ & $\mathrm{OH}$ & $\mathrm{C}_{6} \mathrm{H}_{13}$ & $\mathrm{C}_{6} \mathrm{H}_{13}$ \\
V & $\mathrm{N}\left(\mathrm{CH}_{3}\right)_{2}$ & $\mathrm{H}$ & $\mathrm{H}$ & $\mathrm{H}$ \\
VI & $\mathrm{H}$ & $\mathrm{N}\left(\mathrm{CH}_{3}\right)_{2}$ & $\mathrm{H}$ & $\mathrm{H}$ \\
VII & $\mathrm{N}\left(\mathrm{CH}_{3}\right)_{2}$ & $\mathrm{~N}\left(\mathrm{CH}_{3}\right)_{2}$ & $\mathrm{H}$ & $\mathrm{H}$ \\
\hline
\end{tabular}

FIGURE 1: Structures of 2-(3-coumaroyl)benzopyrylium perchlorate and its derivatives.<smiles>[R]c1cc(O)c(C=O)cc1[R]</smiles><smiles>[R2]c1cc2cc(C(C)=O)c(=O)oc2cc1[R2]</smiles><smiles></smiles>

Figure 2: The pathway of the synthesis of 2-(3-coumaroyl)-benzopyrylium salts.

"acceptor," respectively. It was shown recently that the longwavelength absorption bands of these compounds originate from electronic transitions localised on the BP fragment or from ICT $[7,8]$.

Our previous investigations of CBP solvatochromism showed that the spectral parameters of the dyes are dependent on environmental properties in a complex manner [7]. Consequently, new attempts were made to study the structurespectral properties-medium relationship in greater depth. The synthesis of some new CBP derivatives is described, too. The structures of the CBP perchlorates obtained and investigated are shown in Figure 1.

It can be seen that the investigated CBP derivatives contain electron-donating substituents in either the BP or the $\mathrm{CP}$ fragment or in both fragments, which allows the intensity of ICT to change during excitation or relaxation in the excited and ground states. To increase the solubility of the hydroxylcontaining CBP derivatives in organic solvents, hexyl fragments $\left(\mathrm{R}_{3}\right.$ and/or $\left.\mathrm{R}_{4}\right)$ were inserted in the orthoposition with respect to the $\mathrm{OH}$ groups.

\section{Experimental}

2.1. Synthesis of CBP Perchlorates. The synthesis of CBP perchlorates I and V-VII was first described in [16]. Figure 2 shows a scheme of the synthesis.

To obtain CBP perchlorates, $0.01 \mathrm{~mol}$ of a salicylic aldehyde derivative and an equimolar quantity of a 3-acetylcoumarin derivative were dissolved in $30 \mathrm{~mL}$ concentrated acetic acid. Next, 5-6 mL of $50 \%$ aqueous $\mathrm{HClO}_{4}$ was added. The resulting mixture was refluxed for 10-30 min (see Table 1) and then cooled. Precipitation took place either just after cooling or after the further addition of $5 \mathrm{~mL}$ isopropanol.
The precipitate was filtered, washed with ethanol, and then recrystallised several times from an organic solvent. The purity of the product was tested using an Agilent 1100 HPL chromatograph. The synthesis conditions for each CPB derivative are detailed in Table 1 . The physicochemical characteristics of the products are presented in Table 2.

2.2. Spectral Measurements. Absorption spectra were recorded using a PerkinElmer Lambda35 UV/Vis spectrophotometer, fluorescence spectra were obtained with a Varian Cary Eclipse fluorescence spectrophotometer, and lifetime measurements were carried out using an ISS ChronosFD fluorimeter. Absorption and fluorescence spectra were deconvoluted into individual bands using Spectra Data Lab software [17]. The quantum yields were measured using aqueous solutions of Cyanine $5(\phi=27 \%)$ [14] and Rhodamine B $(\phi=31 \%)$ [11], as well as fluorescein solutions in $0.1 \mathrm{M}$ aqueous $\mathrm{NaOH}(\phi=95 \%)$ [11]. Lifetime was determined using aqueous solutions of Alexa 647 ( $\tau=1.04 \mathrm{~ns})$ [15], Rhodamine 6G ( $\tau=4,08 \mathrm{~ns})$ [13], and fluorescein solutions in $0.1 \mathrm{M}$ aqueous $\mathrm{NaOH}$ ( $\tau=4.1 \mathrm{~ns})$ [12].

2.3. Solvatochromism and Solvatofluorochromism Investigations. The solvatochromic and solvatofluorochromic effects were studied in several steps using 14 solvents (for parameters, see Table 3). Furthermore, the commercial solvents used for the solvatochromism investigations were purified and dried according to [18].

The solubility of each CBP derivative in all the solvents was checked first. As a result, the quantity of solvents used for the investigations of particular CBP perchlorates was lower and varied from 6 to 12 . 
TABLE 1: Conditions for the synthesis of CBP derivatives.

\begin{tabular}{lcccc}
\hline & Refluxing duration, min & Solvent for the initiation of precipitation & Solvents used for recrystallisation & Yield, \% \\
\hline I & $10-15$ & - & Ethanol & 77 \\
II & 20 & - & Ethanol & 73 \\
III & 20 & - & Ethanol & 72 \\
IV & 30 & - & Concentrated acetic acid & 66 \\
V & 20 & Isopropanol & Isopropanol & 70 \\
VI & 20 & Isopropanol & Isopropanol-acetonitrile $(4: 1 \mathrm{v} / \mathrm{v})$ & 64 \\
VII & 20 & & Acetonitrile & 57 \\
\hline
\end{tabular}

TABle 2: Physicochemical parameters of the CBP salts.

\begin{tabular}{|c|c|c|c|c|c|}
\hline & \multirow{2}{*}{ Colour of crystals } & \multirow{2}{*}{ Melting point, ${ }^{\circ} \mathrm{C}$} & \multirow{2}{*}{ Peaks in mass spectra ${ }^{\mathrm{a}}, m / z$} & \multicolumn{2}{|c|}{ Elemental analysis ${ }^{\mathrm{b}}, \%$} \\
\hline & & & & $\mathrm{C}$ & $\mathrm{H}$ \\
\hline I & Greenish black & 291-292 & 275 & $78.50(78.54)$ & $4.11(4.03)$ \\
\hline II & Orange & 278 & 375 & $76.72(76.78)$ & $6.21(6.17)$ \\
\hline III & Dark violet & 255 & $375,850^{c}$ & $76.54(76.78)$ & $6.10(6.17)$ \\
\hline IV & Dark red & 246 & 475 & $75.71(75.76)$ & $7.35(7.42)$ \\
\hline $\mathrm{V}$ & Dark blue & 286 & 346 & $76.32(76.28)$ & $5.88(5.82)$ \\
\hline VI & Dark blue & 275 & 346 & $76.03(76.28)$ & $5.96(5.82)$ \\
\hline VII & Dark blue & $251-252$ & 417 & $74.84(74.79)$ & $6.96(7.00)$ \\
\hline
\end{tabular}

${ }^{a}$ Mass spectra were recorded on a Bruker Daltonics MALDI-TOF MS instrument; ${ }^{b}$ elemental analysis was performed on an Elementar Vario El Cube CHNS analyser; calculated values are given in parentheses; ${ }^{c}$ a peak corresponding to the $\left[2(\mathrm{III}) \cdot \mathrm{ClO}_{4}\right]^{+}$ion.

To prevent the self-association of CBP perchlorates, plots were obtained of optical density versus concentration in all the solvents used. Working concentrations of CBP in the range $3 \times 10^{-6}-8 \times 10^{-6} \mathrm{~mol} / \mathrm{L}$ were taken from the linear sections of the plots.

Since some organic cations demonstrate anomalous spectral behaviour in aromatic or proton-donating solvents, regression analysis of CBP spectral characteristics was initially performed only for aprotic nonaromatic solvents (1-8 in Table 3). Then, depending on the results obtained, such an analysis was carried out for larger groups of solvents-aprotic solvents + alcohols (11-14 in Table 3) or aprotic nonaromatic and aromatic solvents of high polarisability (9-10 in Table 3 ).

Multicomponent regression analysis of the dependence between the spectral characteristics of the CBP derivatives and the solvent parameters was done using Statistica 6.0.

\section{Discussion}

Since CBP electronic transitions can be localised on the "antenna" or "acceptor" fragments, as they are of the ICT type $[7,8]$, the dyes demonstrate a complex spectral behaviour that is highly dependent on the solvent properties. Thus, in aromatic solvents, the positions of the absorption bands undergo bathochromic shifts when in media of higher polarisability [7], because polarisable solvent molecules apparently stabilise cations in the Frank-Condon excited state. At the same time, the influence of the nucleophilicity of aromatic solvents is abnormally weak owing to the formation of $\pi$-complexes between the aromatic molecules and organic cations $[19,20]$. In alcohols or mixtures containing water, some CBP derivatives form hydrogen bonds with the solvent molecules and demonstrate a dependence on the electrophilic properties of the medium.

To prevent the influence of $\mathrm{H}$-bonding and $\pi$-complex formation on the spectral parameters of the CBP derivatives, a group of aprotic solvents of different polarity, polarisability, nucleophilicity, and electrophilicity was initially chosen. Then, if the CBP spectral parameters were found to be dependent on solvent basicity or electrophilicity, investigations for a larger group of solvents, including alcohols, were attempted. When such a dependence of spectral parameters on solvent polarity or polarisability was detected, the investigations were performed for both aprotic and aromatic solvents.

The following spectral characteristics of CBP in the different media were measured: maxima of the long-wavelength absorption bands ( $\nu_{\mathrm{abs}}$ and $\left.\lambda_{\mathrm{abs}}\right)$ and emission bands $\left(\nu_{\mathrm{fl}}\right.$ and $\left.\lambda_{\mathrm{ff}}\right)$, Stokes shifts $\left(\Delta v_{\mathrm{St}}\right)$ and quantum yields $(\phi)$ of fluorescence, and lifetime in the excited state $(\tau)$. All these parameters for I-VII are listed in Tables 1S-7S in the Supplementary Materials available online at http://dx.doi.org/10.1155/2014/ 508153.

The dependence of the spectral characteristics on the solvent properties was studied by regression analysis using two groups of solvent parameters: the Koppel-Palm parameters [9] (Kirkwood constant (polarity) $(Y)$, polarisability $(P)$, nucleophilicity $(B)$, and electrophilicity $(E)$ ) and the KamletTaft parameters [10] (the empirical parameter $\pi^{*}$ (polarity 
TABLE 3: Parameters of the solvents used".

\begin{tabular}{|c|c|c|c|c|c|c|c|c|}
\hline & \multirow{2}{*}{ Solvent } & \multicolumn{4}{|c|}{ Koppel-Palm parameters } & \multicolumn{3}{|c|}{ Kamlet-Taft parameters } \\
\hline & & $Y$ & $P$ & $B$ & $E$ & $\pi^{*}$ & $\beta$ & $\alpha$ \\
\hline 1 & Dichloromethane & 0.422 & 0.203 & 23 & 2.7 & 0.82 & 0.10 & 0.13 \\
\hline 2 & Chloroform & 0.356 & 0.210 & 14 & 3.3 & 0.58 & 0.10 & 0.20 \\
\hline 3 & Dichloroethane-1,2 & 0.431 & 0.210 & 40 & 3.0 & 0.81 & 0.10 & - \\
\hline 4 & Diethyl ether & 0.345 & 0.178 & 283 & 0.1 & 0.27 & 0.47 & - \\
\hline 5 & Tetrahydrofuran & 0.407 & 0.198 & 287 & 0.0 & 0.58 & 0.55 & - \\
\hline 6 & Butanone & 0.461 & 0.187 & 209 & 2.0 & 0.67 & 0.48 & 0.06 \\
\hline 7 & Acetone & 0.465 & 0.180 & 224 & 2.1 & 0.71 & 0.43 & 0.08 \\
\hline 8 & Acetonitrile & 0.480 & 0.175 & 160 & 5.2 & 0.75 & 0.40 & 0.19 \\
\hline 9 & Benzonitrile & 0.471 & 0.236 & 155 & 2.2 & 0.90 & 0.37 & - \\
\hline 10 & Nitrobenzene & 0.479 & 0.242 & 67 & 2.1 & 1.01 & 0.30 & - \\
\hline 11 & Methanol & 0.477 & 0.169 & 218 & 10.6 & 0.60 & 0.66 & 0.98 \\
\hline 12 & Propanol-2 & 0.459 & 0.193 & 230 & 7.4 & 0.40 & 0.80 & 0.69 \\
\hline 13 & Butanol-2 & 0.407 & 0.198 & 231 & 7.1 & 0.60 & 0.55 & - \\
\hline 14 & Undecanol-1 & 0.384 & 0.209 & 242 & 10 & 0.45 & 0.82 & 0.70 \\
\hline
\end{tabular}

${ }^{\#}$ Koppel-Palm parameters [9]: $Y$ : polarity (Kirkwood constant), $P$ : polarisability, $B$ : basicity, and $E$ : electrophilicity. Kamlet-Taft parameters [10]: $\pi^{*}:$ polarity + polarisability, $\beta$ and $\alpha$ : basicity and acidity, respectively.

TABLE 4: Results of regression analysis of the dependence between the positions of the long-wavelength absorption band maxima and the Koppel-Palm and Kamlet-Taft solvent parameters ${ }^{\mathrm{a}}$.

\begin{tabular}{llll}
\hline & Regression equations & $n$ & $R$ \\
\hline I & $\nu_{\text {abs }}=12.78 \times B+20507$ & 6 & 0.897 \\
& $\nu_{\text {abs }}=8362 \times \beta+19461$ & 6 & 0.910 \\
\hline II & $\nu_{\text {abs }}=5167 \times Y+18050$ & 11 & 0.718 \\
\hline III & $\nu_{\text {abs }}=1.93 \times B+18325$ & 9 & 0.831 \\
& $v_{\text {abs }}=-744 \times \pi^{*}+641 \times \beta+18920$ & 9 & 0.937 \\
\hline IV & $v_{\text {abs }}=1283 \times Y+18390$ & 8 & 0.811 \\
\hline V & $\nu_{\text {abs }}=4813 \times Y+0.90 \times B+15742$ & 9 & 0.929 \\
& $v_{\text {abs }}=1368 \times \pi^{*}+865.4 \times \beta+16707$ & 9 & 0.799 \\
\hline VI & $v_{\text {abs }}=8537 \times Y+12010$ & 8 & \\
\hline VII & $\nu_{\text {abs }}=5320 \times Y+0.63 \times B+12650$ & 7 & 0.847 \\
\hline
\end{tabular}

${ }^{a} n$ : quantity of solvents used (this depends on the solubility of CBP perchlorates in the given solvents), $R$ : correlation coefficients. Regression coefficients were checked using the $F$-test.

and polarisability), electrophilicity $(\alpha)$, and nucleophilicity $(\beta))$. The equality of coefficients in the regression equations

$$
\begin{gathered}
x=y Y+p P+b B+e E+c, \\
x=c_{1} \pi^{*}+c_{2} \alpha+c_{3} \beta+c_{0}
\end{gathered}
$$

was checked using the $F$-test. The regression analysis equations obtained are listed in Tables 4 and 5 .

These data showed that a better description of the relationship between the spectral characteristics of CBP and the solvent properties was obtained with the Koppel-Palm parameters. The clearest results were obtained for band positions in the absorption spectra. The equations in Table 4 show that the absorption bands of CBP cations are sensitive to only two parameters $-Y$ and $B\left(\pi^{*}\right.$ and $\left.\beta\right)$. It is worth noting that although changes in the absorption spectra seem to be dependent on both these parameters, most of the regression equations are monoparametric. This means that the influence of one parameter is much weaker than that of another one. We also detected a tendency towards absorption band shifting with changing solvent polarisability, but, in the case of aprotic nonaromatic solvents, the correlation between $P$ and $\nu_{\text {abs }}$ was statistically not significant. Examples of monoand diparametric dependencies are shown in Figures 3(a)$3(\mathrm{c})$.

Table 4 and Figure 3 show that regression coefficients $y$ and $b\left(c_{1}\right.$ and $\left.c_{2}\right)$ in all the equations obtained are positive; hence, the increase in the polarity and the nucleophilicity of the medium results in a hypsochromic shift of the longwavelength absorption band. 


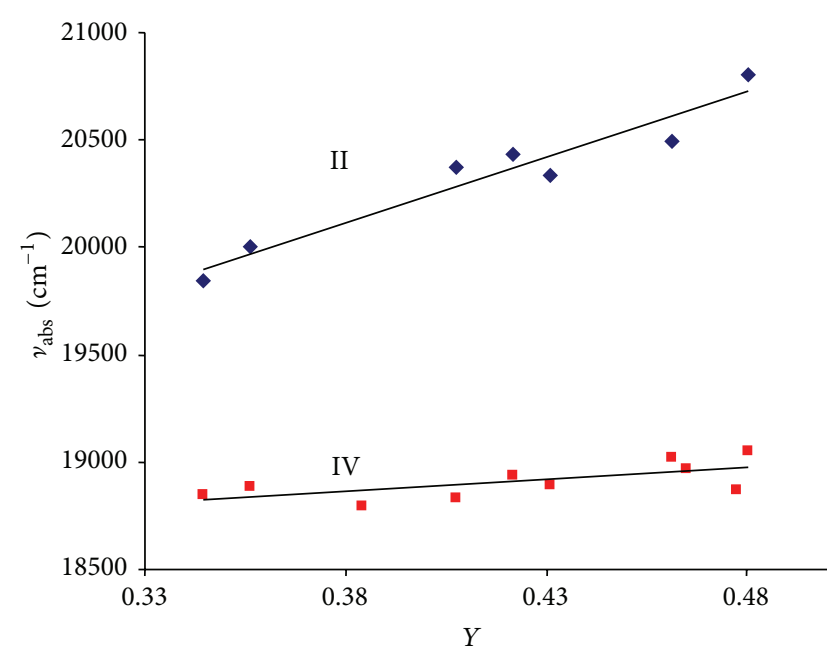

(a)

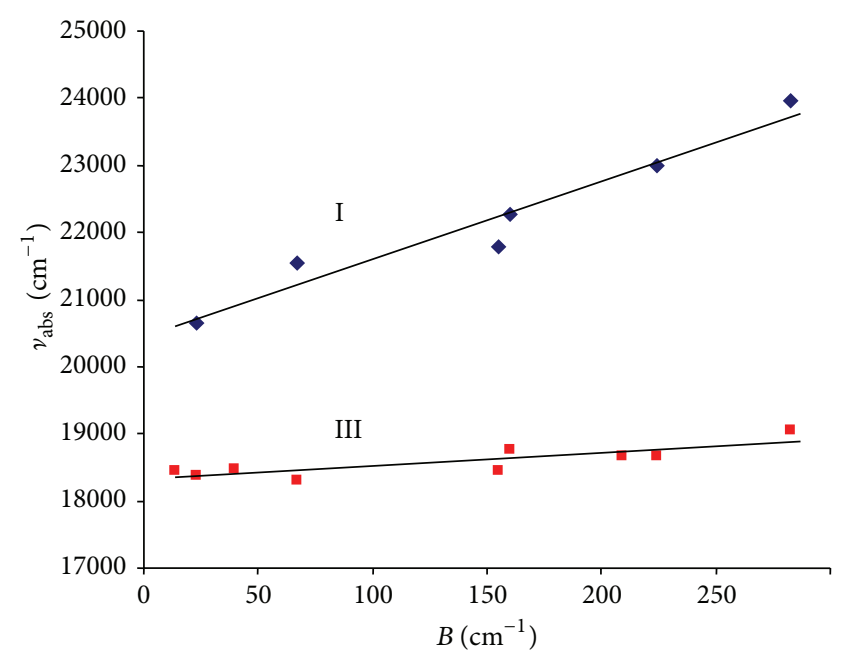

(b)

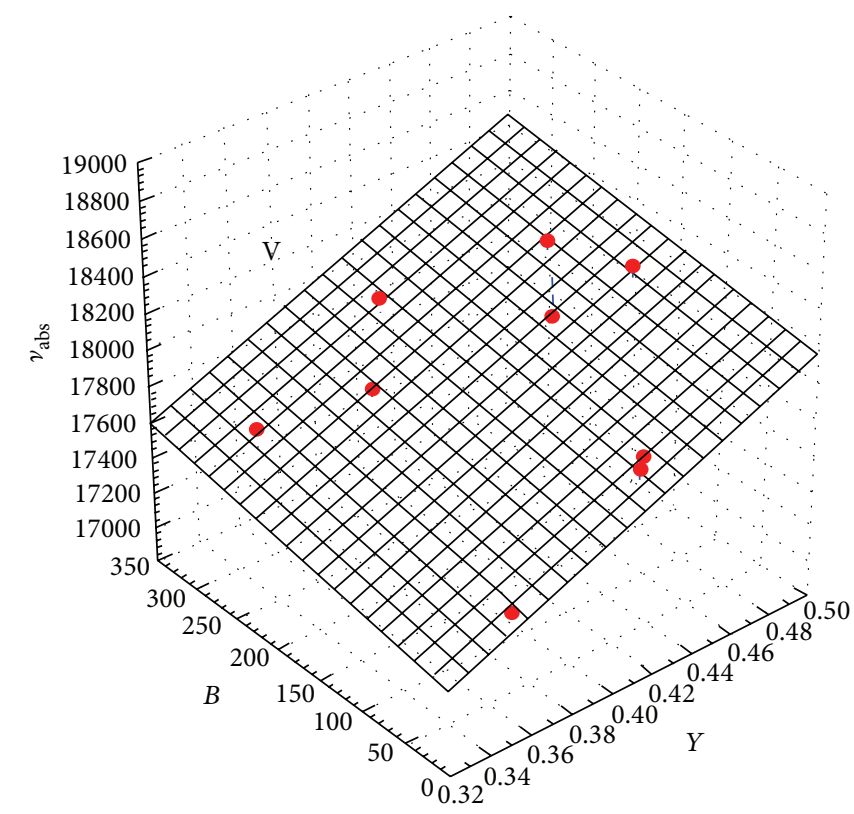

(c)

FIGURE 3: Plots of long-wavelength absorption band maxima $\left(\nu_{\mathrm{abs}}\right)$ against polarity (a), nucleophilicity (b), and both polarity and nucleophilicity (c) of the medium.

Such spectral behaviour can be explained by the redistribution of the positive charge between the $\mathrm{BP}$ and $\mathrm{C}$ fragments in the ground state and by further interfragmental charge transfer following excitation.

Comparison of $v_{\text {abs }}$ values in nucleophilic aliphatic and aromatic solvents shows that the solvent molecules form nucleophilic complexes with the positively charged BP fragment. The structures of such complexes were simulated in $[8,21]$. The larger the positive charge on the BP fragment $\left(q_{\mathrm{BP}}\left(S_{0}\right)\right)$, the stronger the nucleophilic complex.

Interfragmental charge transfer following excitation $\left(\Delta q_{\mathrm{BP}}\left(S_{0} \rightarrow S_{1}^{\mathrm{FC}}\right)\right)$ results in a decrease of positive charge on the $\mathrm{BP}$ fragment owing to the partial delocalisation of the charge on the $\mathrm{C}$ fragment.
When the value of $\Delta q_{\mathrm{BP}}\left(S_{0} \rightarrow S_{1}^{\mathrm{FC}}\right)$ is high, the ICT upon excitation leads to a substantial decrease in the positive charge on the BP fragment and, consequently, to a weakening of the stability of nucleophilic complexes with the solvent molecules.

In this case, the increase in the medium's nucleophilicity can result in a significant lowering of the ground state energy $\left(\Delta E_{S_{0}}\right)$ in comparison with the Franck-Condon excited state $\left(\Delta E_{S_{1}}\right)$ (Figure 4). Thus, in a solvent of higher nucleophilicity, the energy gap between the ground and excited state $\left(E_{S_{0} \rightarrow S_{1}}\right)$ in CBP molecule increases: this explains the hypsochromic shift of the long-wavelength band.

Another phenomenon, also due to ICT following excitation, is the equalisation of the positive charge between 
TABLE 5: Results of regression analysis of the dependence between the fluorescent characteristics and the Koppel-Palm and Kamlet-Taft solvent parameters ${ }^{\mathrm{a}}$.

\begin{tabular}{|c|c|c|c|c|c|}
\hline & \multirow{2}{*}{ Regression equations } & \multirow{2}{*}{$n$} & \multirow{2}{*}{$R$} & \multicolumn{2}{|c|}{ Standard dyes ${ }^{\mathrm{b}}$} \\
\hline & & & & $\varphi$ & $\tau$ \\
\hline I & $\begin{array}{l}v_{\mathrm{fl}}=-4500 \times Y-2.21 \times B+20388 \\
v_{\mathrm{fl}}=-1372 \times \beta+18500\end{array}$ & 6 & 0.996 & {$[11]$} & {$[12]$} \\
\hline II & No dependences & - & - & [11] & [13] \\
\hline III & $\begin{array}{l}v_{\mathrm{fl}}=-8437 \times Y+20370 \\
v_{\mathrm{fl}}=-2036 \times \pi^{*}-1414 \times \beta+18610\end{array}$ & $\begin{array}{l}9 \\
9 \\
\end{array}$ & $\begin{array}{l}0.922 \\
0.901\end{array}$ & {$[11]$} & [12] \\
\hline IV & $\begin{array}{l}v_{\mathrm{fl}}=-9.88 \times B+17754 \\
\tau=-0.01 \times B-0.17 \times E+3.89 \\
\tau=-3.50 \times \beta+3.84\end{array}$ & $\begin{array}{l}8 \\
8 \\
8 \\
\end{array}$ & $\begin{array}{l}0.831 \\
0.973 \\
0.894 \\
\end{array}$ & {$[11]$} & [13] \\
\hline $\mathrm{V}$ & $\begin{array}{l}\varphi=-6.14 \times Y+3.15 \\
\tau=-0.002 \times B+2.803\end{array}$ & $\begin{array}{l}8 \\
6 \\
\end{array}$ & $\begin{array}{l}0.712 \\
0.919 \\
\end{array}$ & {$[14]$} & {$[15]$} \\
\hline VI & $\begin{array}{l}\nu_{\mathrm{fl}}=-2114 \times Y+15315 \\
\nu_{\mathrm{fl}}=-701.3 \times \pi^{*}-531.0 \times \beta+15056 \\
\varphi=-147.50 \times Y+73.43 \\
\tau=-6.49 \times Y+5.34\end{array}$ & $\begin{array}{l}8 \\
8 \\
10 \\
11 \\
\end{array}$ & $\begin{array}{l}0.764 \\
0.870 \\
0.922 \\
0.838 \\
\end{array}$ & {$[14]$} & [15] \\
\hline VII & $\begin{array}{l}\varphi=-3.15 \times E+56.07 \\
\tau=-9.01 \times Y-0.0034 \times B+7.25\end{array}$ & $\begin{array}{l}7 \\
7\end{array}$ & $\begin{array}{l}0.995 \\
0.931\end{array}$ & {$[14]$} & [15] \\
\hline
\end{tabular}

${ }^{a}$ All the designations are given in the text and in Table 4 .

${ }^{\mathrm{b}}$ Publication references [11-15] concerning standard dyes used for the determination of quantum yields and fluorescence lifetimes.

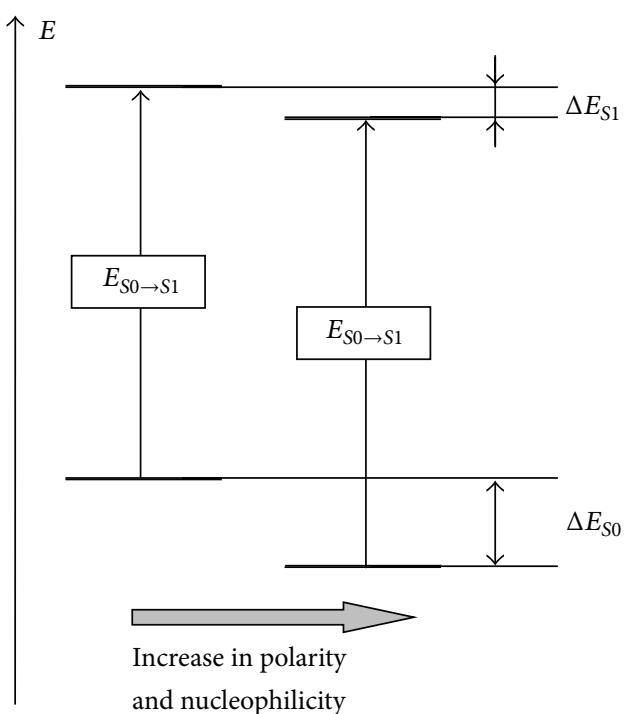

FIGURE 4: The energetics of the ground and excited states in media of greater polarity and nucleophilicity.

the fragments that results in a decrease in the dipole moment. Since ICT leads to depolarisation of the CBP molecules, the increase in polarity of the medium can stabilise CBP more strongly in a more polar ground state than in a less polar excited state. Thus, in a more polar solvent, the energy gap $E_{S_{0} \rightarrow S_{1}}$ between the ground and excited states also increases, which results in a hypsochromic shift of the absorption bands.

If the effect of weakening the stability of nucleophilic complexes upon excitation is stronger than the depolarisation effect (I, III), then the dependence $v_{\mathrm{abs}}=b B+c$ takes place.
In the opposite case (II, VI), the dependence $\nu_{\mathrm{abs}}=y Y+c$ holds. If these effects are of similar intensity, the behaviour of the long-wavelength band can be described by the twoparametric equation $v_{\mathrm{abs}}=y Y+b B+c$.

In the case of CBP derivatives with low $\Delta q_{\mathrm{BP}}\left(S_{0} \rightarrow S_{1}^{\mathrm{FC}}\right)$, there are no dramatic changes in the positive charge on the BP fragment upon excitation. That is why the weakening stability of nucleophile complexes and depolarisation following excitation are less intensive. As a result, the dependencies between $v_{\text {abs }}$ and $B$ or $Y$ are weaker: the regression coefficients $b$ and/or $y$ are lower than for other CBP derivatives.

The relaxation processes in the excited state are accompanied by additional ICT, and the values of the positive charge on $\mathrm{BP}$ and $\mathrm{C}$ fragments in the relaxed excited state differ from those in the Frank-Condon excited state [22]. The spectral behaviour of the CBP emission bands in media of a different nature is not therefore similar to that of the absorption bands. The data listed in Table 5 show that solvatofluorochromic effects are substantially weaker than the solvatochromic ones. This can be explained by the lower positive charge on the $\mathrm{BP}$ fragment in the relaxed excited state. The positions of the emission band maxima of II, V, and VII do not depend on the solvent properties; the band maxima of III and VI undergo bathochromic shifts with increasing medium polarity (Figure 5(a)). Linear dependence between $\nu_{\mathrm{fl}}$ and $B$ was found only for I and IV (Figure 5(b)).

Moreover, the increase in nucleophilicity resulted in the opposite spectral effects: a bathochromic shift was detected for I and a hypsochromic one for IV. This could mean that the positive charge on the BP under radiation deactivation decreases in the case of I and increases in the case of IV.

The quantum yield values of I and oxygen-containing derivatives vary over a large range (Table 5) but they do not 


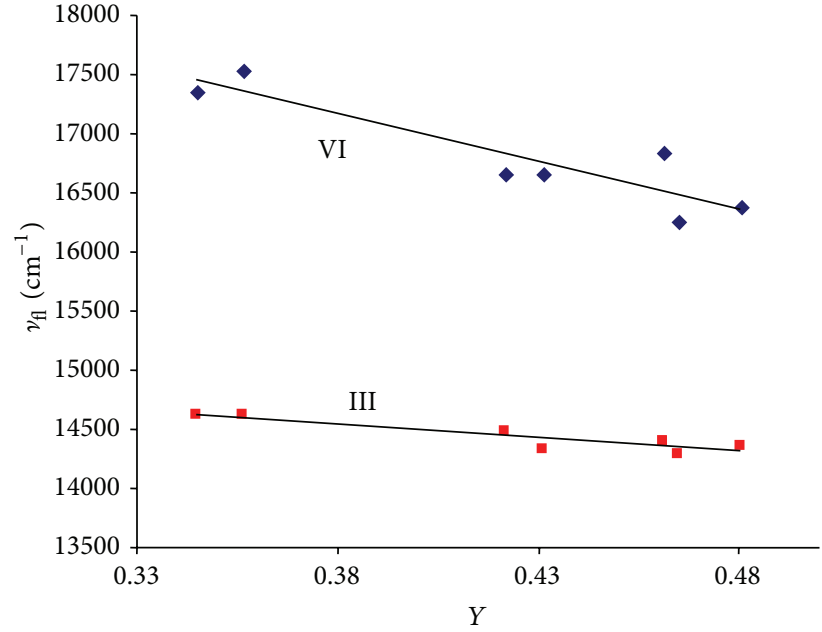

(a)

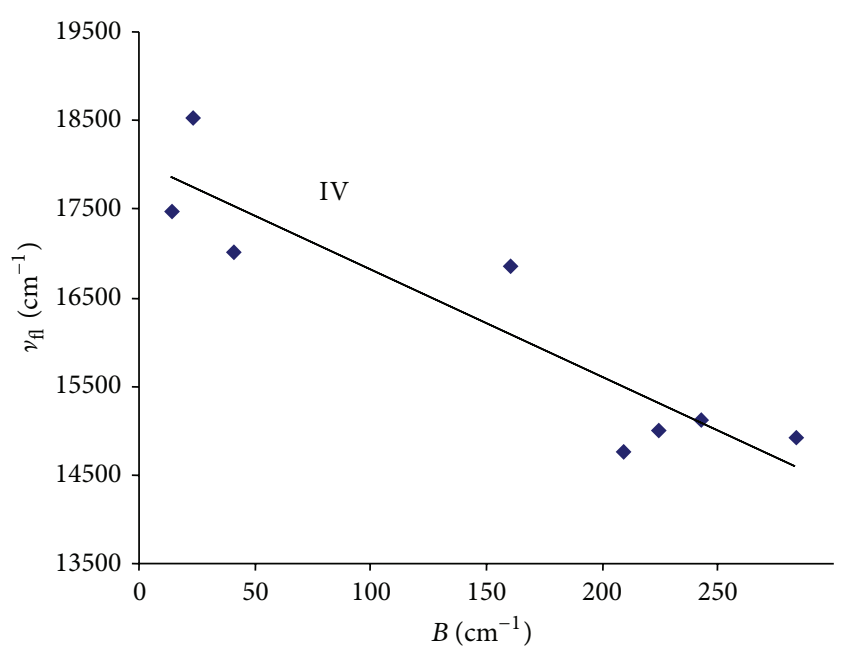

(b)

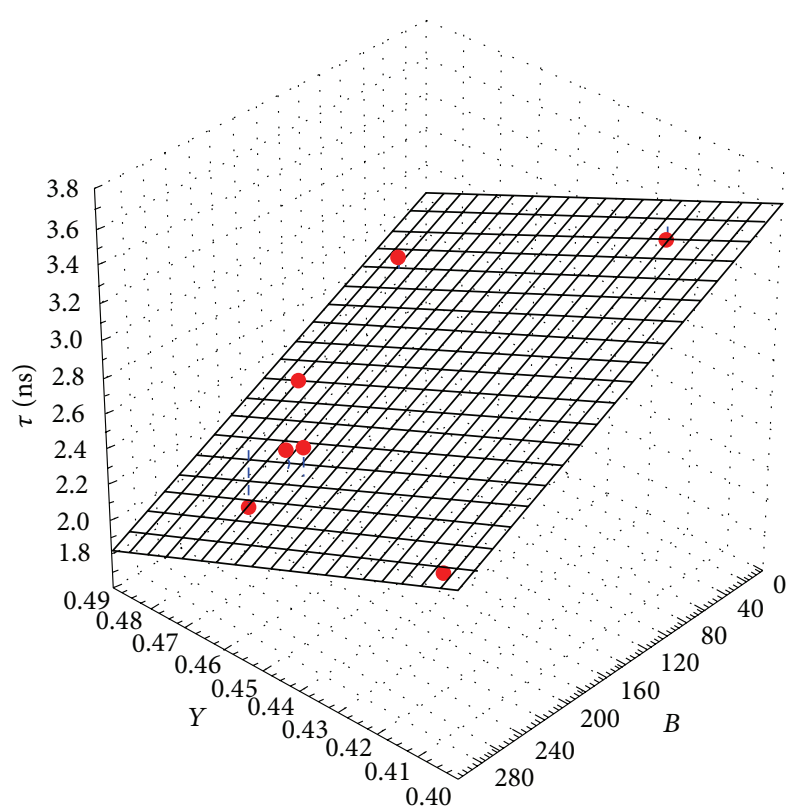

(c)

Figure 5: Plots of emission band maxima $\left(v_{\mathrm{fl}}\right)$ against polarity (a) and nucleophilicity (b). A plot of lifetime value $(\tau)$ against both polarity and nucleophilicity (c) of the medium.

depend on the solvent parameters used in the regression analysis procedure.

The dimethylamino derivatives $\mathrm{V}$ and VI demonstrate the dependence of $\phi$ values on the solvent polarity and VII on the electrophilicity of the medium. In all the cases, an increase in the $Y$ or $E$ parameters of the solvents results in fluorescence quenching.

The emission lifetime of CBP is also a fluorescent parameter sensitive to changes of medium properties (except III, the $\tau$ value of which does not depend on solvent characteristics). The data listed in Table 5 and depicted in Figure 5(c) show the dependence of lifetimes on the solvent polarity and nucleophilicity. Any increase in these parameters leads to a decrease of lifetimes.

\section{Conclusions}

Analysis of solvatochromic and solvatofluorochromic effects of the CBP derivatives showed that the excitation and fluorescent deactivations of these dyes are connected with interfragmental charge transfer, which influences the depolarisation of the molecules and the changes in the relative stability of the nucleophile complexes with the solvent molecules. Therefore, the spectral properties of CBP depend on the polarity and/or nucleophilicity of the solvents.

Some spectral characteristics of CBP, such as the positions of the long-wavelength absorption bands, fluorescent quantum yields, and lifetimes, can be used for testing the medium's properties. However, the clearest information can be obtained 
by analysis of changes in CBP absorption spectra, where the maximum solvatochromic effect was detected.

\section{Conflict of Interests}

The authors declare that there is no conflict of interests regarding the publication of this paper.

\section{Acknowledgments}

The financial support of this work by Ukrainian State Grant 0112 U007568 is gratefully acknowledged. The authors gratefully acknowledge the Physical Chemistry Department of the University of Gdansk (Professor J. Błażejowski and Professor K. Krzymiński) for assistance with testing the structures of CBP using mass spectrometry and elemental analysis.

\section{References}

[1] M. Mandalà, G. Serck-Hanssen, G. Martino, and K. B. Helle, "The fluorescent cationic dye rhodamine $6 \mathrm{G}$ as a probe for membrane potential in bovine aortic endothelial cells," Analytical Biochemistry, vol. 274, no. 1, pp. 1-6, 1999.

[2] B. Ehrenberg, V. Montana, M.-D. Wei, J. P. Wuskell, and L. M. Loew, "Membrane potential can be determined in individual cells from the nernstian distribution of cationic dyes," Biophysical Journal, vol. 53, no. 5, pp. 785-794, 1988.

[3] S. Minegishi, S. Kobayashi, and H. Mayr, "Solvent nucleophilicity," Journal of the American Chemical Society, vol. 126, no. 16, pp. 5174-5181, 2004.

[4] S. Haldar and A. Chattopadhyay, "Hydration dynamics of probes and peptides in captivity," in Reviews in Fluorescence 2010, vol. 2010, pp. 155-172, Springer, New York, NY, USA, 2012.

[5] "Probe design and chemical sensing," in Topics in Fluorescence Spectroscopy, J. R. Lakowicz, Ed., vol. 4, Plenum Press, New York, NY, USA, 1994.

[6] A. P. Demchenko, Introduction to Fluorescence Sensing, Springer Science \& Business Media B.V., Dordrecht, The Netherlands, 2009.

[7] E. V. Sanin, A. I. Novikov, and A. D. Roshal, "Investigations of solvatochromism of 2-(3-coumaroyl)-benzopyrylium dye and its di-substituted derivatives," Functional Materials, vol. 20, no. 3, pp. 366-372, 2013.

[8] E. V. Sanin, A. I. Novikov, and A. D. Roshal, "QuantumChemical Investigations of the Structure and Spectral Characteristics of 2-(3-coumaroyl) benzopyrylium cations," Chemistry of Heterocyclic Compounds, vol. 50, pp. 371-378, 2014.

[9] C. Reichardt and T. Welton, Solvents and Solvent Effects in Organic Chemistry, Wiley-VCH, Weinheim, Germany, 2011.

[10] P. G. Jessop, D. A. Jessop, D. Fu, and L. Phan, "Solvatochromic parameters for solvents of interest in green chemistry," Green Chemistry, vol. 14, no. 5, pp. 1245-1259, 2012.

[11] J. R. Lakowicz, Principles of Fluorescence Spectroscopy, Kluwer Academic/Plenum Publishers, New York, NY, USA, 2nd edition, 1999.

[12] D. Magde, G. E. Rojas, and P. G. Seybold, "Solvent dependence of the fluorescence lifetimes of xanthene dyes," Photochemistry and Photobiology, vol. 70, no. 5, pp. 737-744, 1999.
[13] N. Boens, W. Qin, N. Basarić et al., "Fluorescence lifetime standards for time and frequency domain fluorescence spectroscopy," Analytical Chemistry, vol. 79, no. 5, pp. 2137-2149, 2007.

[14] R. B. Mujumdar, L. A. Ernst, S. R. Mujumdar, C. J. Lewis, and A. S. Waggoner, "Cyanine dye labeling reagents: sulfoindocyanine succinimidyl esters," Bioconjugate Chemistry, vol. 4, no. 2, pp. 105-111, 1993.

[15] V. Buschmann, K. D. Weston, and M. Sauer, "Spectroscopic study and evaluation of red-absorbing fluorescent dyes," Bioconjugate Chemistry, vol. 14, no. 1, pp. 195-204, 2003.

[16] P. Czerney and H. Hartmann, "Benzopyryliumsalze, IV. Darstellung und spektrale Eigenschaften von 2-Cumar-3'-yl benzopyryliumsalzen," Journal für Praktische Chemie, vol. 325, pp. 505-510, 1983.

[17] A. O. Doroshenko, Spectral Data Lab Software, Institute of Chemistry at Kharkiv National University, Kharkiv, Ukraine, 1999.

[18] A. Weissberger, E. S. Proskauer, J. A. Riddick, and E. E. Toops, "Organic solvents. Physical properties and methods of purification," in Technique of Organic Chemistry, A. Weissberger, Ed., vol. 7, Interscience Publishers, New York, NY, USA, 1955.

[19] A. A. Ishchenko, V. A. Svidro, and N. A. Derevyanko, "Solvatofluorochromy of cationic cyanine dyes," Dyes and Pigments, vol. 10, no. 2, pp. 85-96, 1989.

[20] M. Wähnert, S. Dähne, and R. Radeglia, "The effect of solvents on the electronic structure of symmetrical polymethinecyanines ${ }^{1} \mathrm{H}$ - and ${ }^{13} \mathrm{C}$-NMR spectroscopic investigations," Advances in Molecular Relaxation and Interaction Processes, vol. 11, pp. 263-282, 1977.

[21] A. D. Roshal, D. Y. Minayev, Y. F. Pedash, and A. I. Novikov, "Spectral properties of phenylbenzopyrylium salts in solutions as a result of their nucleophilic solvation," Polish Journal of Chemistry, vol. 76, no. 9, pp. 1301-1321, 2002.

[22] E. V. Sanin, A. I. Novikov, and A. D. Roshal, "Investigations of bis-chromophore systems: relationship between spectral behaviour and charge transfer in 2-(3-coumaroyl)-benzopyrylium perchlorates," French-Ukrainian Journal of Chemistry, vol. 1, pp. 143-147, 2013. 

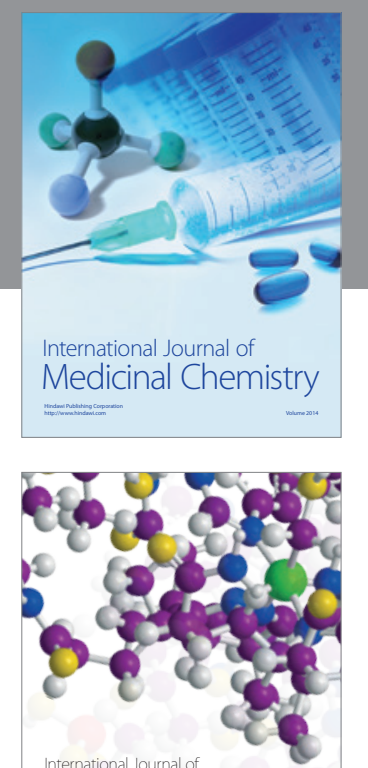

\section{Carbohydrate} Chemistry

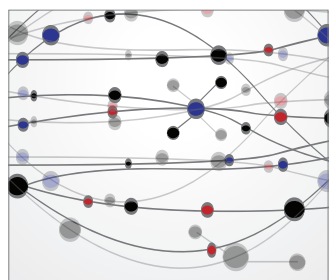

The Scientific World Journal
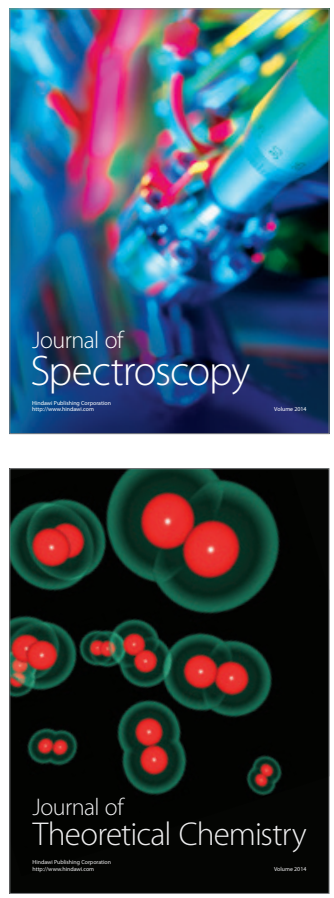
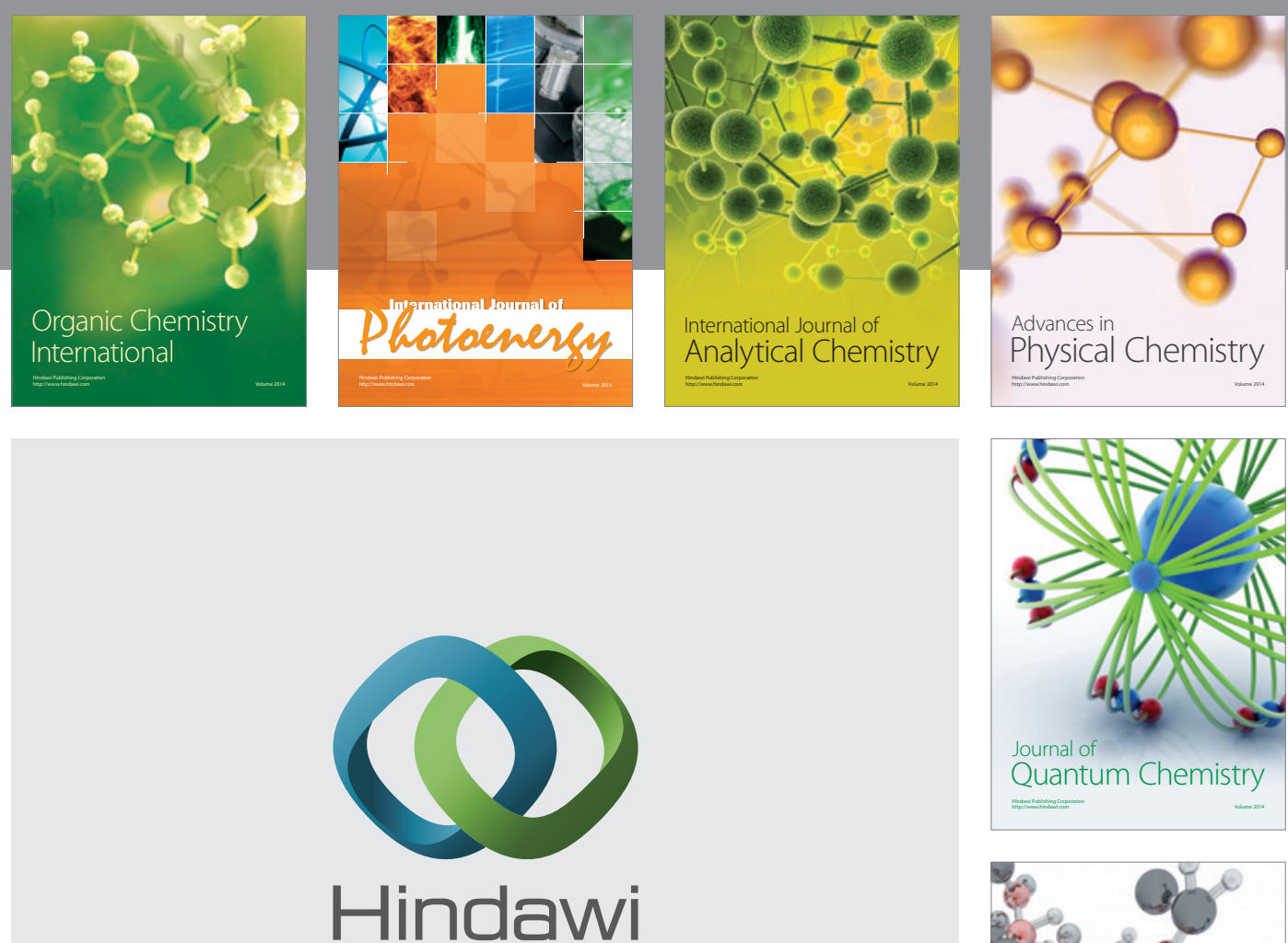

Submit your manuscripts at

http://www.hindawi.com

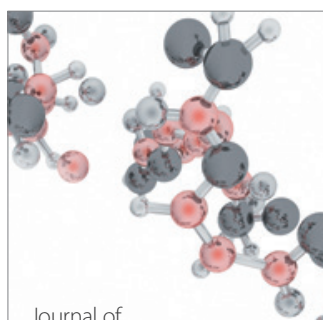

Analytical Methods

in Chemistry

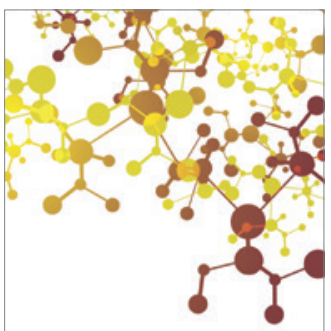

Journal of

Applied Chemistry

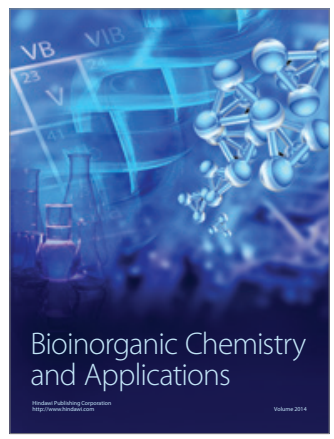

Inorganic Chemistry
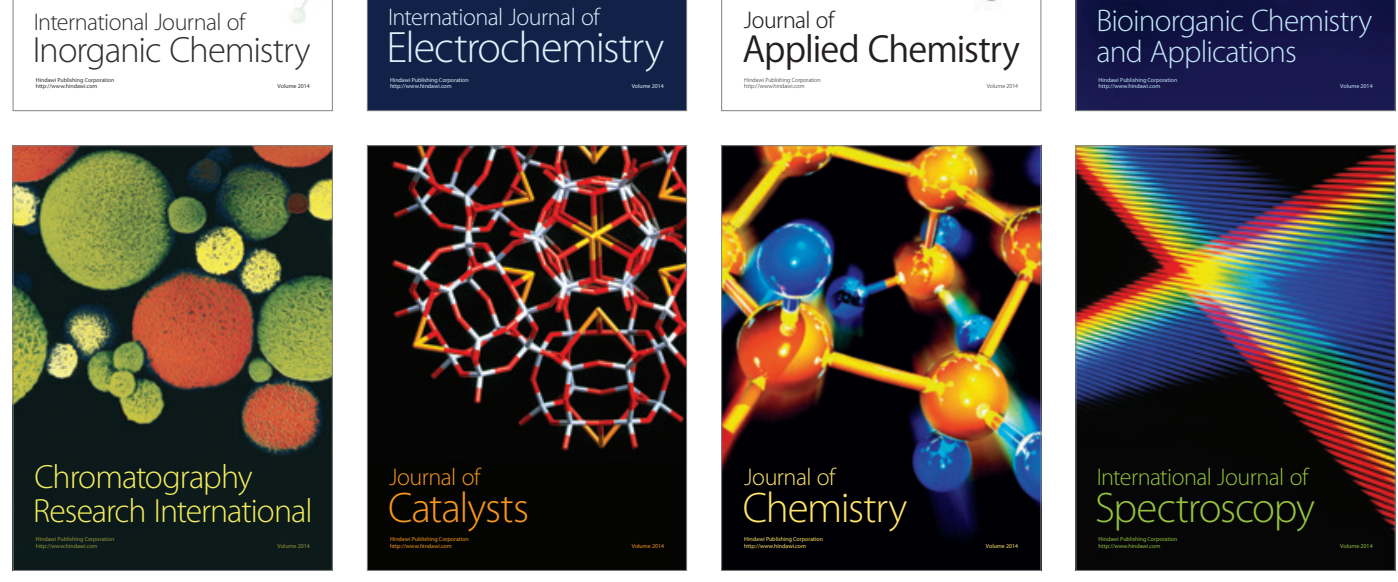